\title{
L'UNIVERSITÀ ITALIANA NEL NOVECENTO. NUOVI ITINERARI STORIOGRAFICI E INEDITI PERCORSI DI RICERCA
}

Por Luigiaurelio Pomante. Milano: FrancoAngeli, 2020, 205 pages. ISBN 978-88917-8980-8

Tra gli anni Ottanta e Novanta del secolo scorso, le ricerche sulla storia dell'istruzione superiore e delle università hanno acquisito nuovi e importanti stimoli dal contributo e dall'operato di centri e gruppi di ricerca specialistici, che si sono fatti promotori di interessanti seminari e incontri di studio, i quali hanno di fatto incentivato e arricchito l'interesse del mondo accademico in merito alla storia dell'Università.

Nell'ultimo trentennio è stata così inaugurata un'inedita e fiorente stagione di studi sull'istruzione superiore caratterizzata, nello specifico, da un maggiore rigore metodologico e da un incremento delle ricerche relative alle politiche e agli ordinamenti universitari in epoca contemporanea.

In linea con questo rinnovamento operativo e tematico, che contraddistingue ancora oggi il dibattito storiografico sulle università e sull'istruzione superiore, si pone il recente volume di Luigiaurelio Pomante, L'Università italiana nel Novecento. Nuovi itinerari storiografici e inediti percorsi di ricerca.

Il testo, edito da FrancoAngeli, si fa apprezzare soprattutto per la peculiare sensibilità storiografica con cui l'Autore ha condotto la sua indagine: sulla scorta di una larga messe di fonti archivistiche e a stampa, il lavoro mette in luce filoni di ricerca finora scarsamente o per nulla approfonditi e si propone di suggerire innovative interpretazioni a temi e nodi cruciali, che hanno definito la storia universitaria italiana nel corso del Novecento. 
Il presente volume, grazie ad una originale impostazione di fondo e alla varietà dei temi trattati, si discosta dalla maggior parte delle precedenti monografie pubblicate dall'Autore, ma si colloca in sostanziale continuità con il suo interesse verso tematiche innovative e con l'uso di fonti in gran parte inedite, elementi che valorizzano la sua ricerca in ambito internazionale e che, in generale, consentono di favorire la comprensione dell'evoluzione e della complessa organizzazione del sistema universitario italiano.

Il volume si apre con un ampio capitolo dedicato all'ambizioso progetto del regime fascista di procedere con il recupero e con la ridefinizione della gloriosa tradizione accademica italiana: nello scenario di totalitaria modernizzazione avviata nel corso del Ventennio, l'Università italiana rivestiva, infatti, un ruolo pioneristico di rinnovamento culturale, scientifico e sociale; essa era la fucina della futura classe dirigente del Paese e, in quanto tale, era il luogo predefinito a 'plasmare' il modello del così detto «uomo nuovo» fascista.

Le università, che avevano naturalmente aderito ai condizionamenti politici e intellettuali del regime, erano chiamate ad affermare il loro 'primato' in ambito scientifico e a trasformarsi in luoghi di elaborazione della cultura fascista, la quale doveva necessariamente essere sorretta da una solida base teorica e da una rigorosa formazione etico-politica.

La categoria storiografica dell' «invenzione della tradizione», già teorizzata da E. Hobsbawm e T. Ranger negli anni Ottanta del Novecento, viene qui applicata da Pomante all'analisi del processo di ricostruzione ideologica dell'identità nazionale, voluto fortemente da Mussolini e dal suo regime, un'operazione che passava automaticamente anche attraverso il recupero delle «nobili tradizioni» e della "gloria intellettuale» dell'Accademia italiana, e che finì per tratteggiare una vera e propria «religione politica», officiata e ribadita nel corso di innumerevoli manifestazioni e adunanze.

Questa «invenzione della tradizione» leggendaria dei primi Atenei italiani si prefigurava come lo strumento del regime atto ad individuare un evento mitico, con cui segnare la nascita, o meglio la ri-nascita, del popolo italiano. 
Un 'inedito' percorso di ricerca, come suggerisce il sottotitolo del volume, viene offerto dall'Autore nel secondo capitolo, dedicato alle pubblicazioni periodiche italiane per professori universitari, che si fa apprezzare, in modo particolare, per l'abilità e la sistematicità con cui tali fonti a stampa sono state interrogate.

Questa tipologia di riviste ha destato l'attenzione di alcuni studiosi dell'istruzione superiore solo in tempi recenti: nell'ambito della storia dell'Università, infatti, erano state finora analizzate specialmente riviste studentesche e goliardiche e, contrariamente a quanto accaduto ai periodici magistrali e per la Scuola, esse non erano state debitamente prese in considerazione.

Le riviste professorali costituiscono certamente una componente piuttosto variegata della stampa periodica universitaria ma, se opportunamente indagate, esse si rivelano una fonte indispensabile alla ricostruzione storica delle caratteristiche del sistema universitario italiano.

Pur essendo generalmente espressione delle idee e delle posizioni dei singoli docenti di ruolo, questa tipologia di pubblicazioni fungeva da strumento di comunicazione interna al mondo accademico e da portavoce delle problematiche e delle questioni universitarie di rilevante interesse nazionale.

Escludendo i numerosi periodici universitari 'minori', nati come occasionali esperienze editoriali di singole sedi accademiche, l'Autore fissa correttamente la data di avvio delle pubblicazioni nazionali rivolte ai professori universitari con la prima uscita de L'Università. Rivista dell'istruzione superiore pubblicata da una società di professori, edita a Bologna tra il 1887 e il 1891.

A tale prima esperienza nazionale fecero seguito altre numerose riviste, tra cui Pomante individua e descrive le più significative e importanti, ovvero: L'Università italiana, edito a Bologna a partire dal 1902, Gli Annali della Università d'Italia. Rivista bimestrale dell'istruzione superiore, pubblicati per la prima volta il 29 ottobre 1939 a cura del ministero dell'Educazione Nazionale, L'Università italiana. Quindicinale di informazioni universitarie, pubblicato dall'Università di Roma a partire dal $1^{\circ}$ aprile 1946, e infine Il Giornale dell'università. Organo degli atenei d'Italia, testata ufficiale dell'ANPUR, nata nel gennaio 1951. 
Questi periodici professorali ponevano l'accento sui pregi e difetti della poliedrica realtà accademica italiana, la quale aveva necessariamente subìto l'influenza dei complessi eventi storici e bellici che segnano la storia della nostra Penisola.

Oltre a proporre nuove sfide da intraprendere in ambito didattico-scientifico, infatti, le riviste misero in luce le principali criticità del sistema d'istruzione superiore italiano, problematiche che si erano d'altronde acuite nel periodo post-bellico, quando l'Università dovette fare i conti con il processo di epurazione dall'ideologia fascista e con una moltitudine di studenti interessati più alla conclusione che alla qualità dei loro studi.

A ciò si aggiungevano ovviamente le difficili condizioni materiali e strutturali delle università, e la dissoluzione degli apparati amministrativi e burocratici.

L'analisi delle problematiche legate all'ambiente accademico italiano del secondo dopoguerra continua nel terzo capitolo del volume, in cui l'Autore punta il focus della sua attenzione al «forte disagio materiale e morale» in cui all'epoca versavano i docenti universitari.

Ancora una volta, i periodici professorali si fecero interpreti delle criticità dell'ambiente accademico e dell'animato dibattito scaturitosi sullo status giuridico, economico e culturale dei professori italiani, una disputa che sfociò anche in numerose richieste fatte pervenire al ministero della Pubblica Istruzione tramite le associazioni sindacali.

I professori universitari ribadivano con estrema fermezza «l'assoluta indipendenza» del loro ruolo dai poteri dello Stato, che avrebbero potuto altrimenti limitare la loro libertà scientifica e intellettuale, e auspicavano modifiche significative nel processo di reclutamento accademico.

Nonostante la difficile situazione economica del Paese, il ministero, e nello specifico il ministro Guido Gonella, salito al dicastero della Minerva nel luglio 1946, volle dare un segnale di vicinanza alla categoria dei professori universitari, riconoscendo la validità delle loro rivendicazioni, promettendo una maggiore rapidità negli avanzamenti di carriera e un miglioramento della condizione economico-giuridica dei docenti. 
Il quarto capitolo de L'Università italiana nel Novecento torna a occuparsi della stampa periodica per professori universitari, questa volta però lo fa attraverso l'analisi di un campione di circa tremila necrologi di docenti, apparsi nelle più importanti riviste nazionali dedicate all'istruzione superiore dell'età contemporanea. Una fonte, i necrologi, che per lungo tempo è stata completamente trascurata dalla storiografia educativa e universitaria, ma che ha, invece, rivelato una grande potenzialità euristica: essa è, infatti, in grado di restituirci l'immagine 'reale' e una visione forse più 'umana' del complesso sistema accademico italiano.

Attraverso questa particolare e innovativa tipologia di fonte, con grande coerenza metodologica, l'Autore prova a ricostruire l'evoluzione della figura e del ruolo del docente universitario, andando al di là della mera natura celebrativa dei componimenti e provando a scioglierne le istanze culturali, ideologiche, pedagogiche ed educative.

I necrologi presi in esame, pubblicati tra il 1880 e il 1960, permettono di fatto di individuare le caratteristiche principali delle condizioni giuridico-economiche e culturali della classe dei professori universitari, e di delinearne gli orientamenti politici e scientifici.

Il capitolo mette bene in luce come tali necrologi fossero apprezzabili per la ricchezza di informazioni e per la pluralità di riferimenti: i componimenti non erano mai privi di rimandi al contesto storico-sociale e miravano a evidenziare la qualità della produzione scientifica e della «dimensione intellettuale e morale» del docente defunto, non tanto quindi la sua posizione accademica, quanto la rilevanza in un determinato ambito scientifico.

Le rubriche dedicate ai necrologi avevano sicuramente una vera e propria valenza pedagogica, in quanto restituivano al 'giovane' mondo universitario modelli e ideali di «condotta professionale», ma non mancavano di dare voce e risonanza anche alle problematiche e alle criticità tipiche dell'ambiente accademico italiano d'età contemporanea.

Il volume si chiude, infine, con un ulteriore elemento di pregio, ovvero un capitolo dedicato ai musei per la storia dell'Università, una particolare tipologia di istituzione universitaria purtroppo ancora oggi scarsamente considerata, al contrario meritevole di grande interesse, al pari dei già noti musei dell'educazione o della scuola. 
In linea con i più recenti orientamenti della ricerca storico-educativa, che auspicano e incentivano la conservazione del patrimonio storico-educativo e la valorizzazione dei beni culturali scolastici, Pomante si sofferma quindi sulla descrizione delle uniche due strutture museali per l'Università presenti in Italia, vale a dire il Museo per la Storia dell'Università di Pavia e il Museo Europeo degli Studenti (MEUS) di Bologna; due realtà molto diverse tra loro, sia dal punto di vista tematico sia dal punto di vista 'cronologico', che rispondono però al comune «progetto di musealizzazione» del vasto patrimonio oggettuale, archivistico-librario, iconografico e fotografico della storia dell'Università.

Il primo dei due musei, quello dell'Università di Pavia, ha origine nel 1932, quando a seguito della mostra allestita dal prof. Antonio Pensa per celebrare la memoria dell'anatomista e accademico Antonio Scarpa, in occasione del centenario della sua morte e del IV Congresso della Società Italiana di Anatomia, l'allora rettore, il prof. Ottorino Rossi, decise di dotare la sede pavese di una struttura museale permanente.

L'iniziativa, coerente con gli obiettivi del 'glorioso' progetto fascista di recupero e valorizzazione della tradizione accademica italiana, si proponeva di conservare il patrimonio storico-culturale dell'Ateneo e di permetterne la fruizione a un pubblico il più possibile ampio.

Nel corso del tempo il Museo, ubicato nel palazzo centrale dell'Università e inaugurato ufficialmente nel 1936, ha beneficiato di numerose e consistenti donazioni, e si è trasformato in un profittevole centro di studio e ricerca.

L'altra sede museale, ovvero il MEUS di Bologna, è un'istituzione più recente (2009) ed ha un carattere meno istituzionale rispetto a quella di Pavia, ciò nonostante rappresenta una vera e propria eccezione tra le realtà museali europee.

Pomante spiega bene come il Museo abbia di fatto l'obiettivo di «favorire la conoscenza dei mutamenti inerenti la figura dello studente nel corso dei secoli», mettendo in luce numerosi aspetti della vita studentesca, come l'associazionismo, l'impegno politico, la questione di genere e l'accesso all'Università, le attività sportive e goliardiche o la mobilità.

Il MEUS, ospitato all'interno di Palazzo Poggi, nasce e si sviluppa grazie all'incontro tra associazioni di ex studenti e alcuni storici dell'istruzione 
superiore, primo fra tutti il prof. Brizzi, oggi segretario generale del Centro Interuniversitario per la Storia delle Università Italiane (CISUI) e direttore dell'Archivio storico dell'Università di Bologna.

Entrambi i musei svolgono un importante ruolo nel contesto accademico italiano: oltre ad inserirsi nello scenario della vagheggiata terza missione universitaria, promossa sia dal MIUR sia dalle singole sedi universitarie, essi alimentano l'impegno alla valorizzazione della tradizione accademica del nostro Paese, al fine di recuperare quel prestigioso passato che caratterizza la storia degli atenei italiani.

L'auspicio dell'Autore, che fa da eco anche a quello di altri studiosi dell'istruzione superiore, è quello di poter finalmente riscoprire l'ingente e significativo patrimonio letterario, storico, scientifico e artistico delle università, purtroppo ancora oggi mal conservato o non adeguatamente stimato.

In conclusione, L'Università italiana nel Novecento. Nuovi itinerari storiografici e inediti percorsi di ricerca fornisce alla storia dell'istruzione superiore e delle università un contributo davvero innovativo e significativo, e si fa apprezzare per l'estrema sensibilità e coerenza della ricostruzione storiografica.

Le molteplici e differenti fonti archivistiche e a stampa, prese in esame con grande rigore metodologico, hanno permesso all'Autore di analizzare in modo critico i cambiamenti subìti dalle università italiane, e di valorizzare la ricchezza e l'eterogeneità del loro patrimonio storico-educativo e scientifico.

Nell'ambito del rinnovamento della storiografia universitaria nazionale, avviato ormai da più di trent'anni e oggi quantomai attivo e aperto alle nuove istanze internazionali, il volume ha inoltre il merito di aver presentato una pluralità di questioni finora poco o per nulla approfondite, che hanno tratteggiato i lineamenti della realtà accademica italiana tra Otto e Novecento, e di aver messo a disposizione chiavi di lettura originali e stimolanti, che forniscono all'intera comunità scientifica importanti spunti di riflessione.

Sofia Montecchiani

Università degli Studi di Macerata s.montecchiani2@unimc.it 